\title{
Compostos voláteis associados ao risco carcinogênico e não carcinogênico
}

\author{
Volatile compounds associated with carcinogenic and non-carcinogenic risk
}

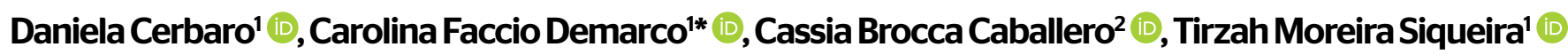

口

\begin{abstract}
RESUMO
Opresentetrabalho consistena avaliação da existência de risco esua quantificação em casos de vazamentos de derivados do petróleo em uma refinaria localizada na Região Sudeste do Brasil. A área apresenta solo subsuperficial e água subterrânea contaminados por hidrocarbonetos de petróleo e outras substâncias em consequência do funcionamento de uma refinaria. Foram consideradas as vias de exposição por inalação aos contaminantes presentes nesses dois meios. A quantificação do risco de câncer e do quociente de perigo foi realizada para cada substância química, permitindo verificar que, para a exposição a água subterrânea contaminada, não houve efeito carcinogênico e não carcinogênico para os compostos analisados por meio da via de inalação. Já para a exposição a solo subsuperficial contaminado, verificou-se que todos os elementos detectados apresentaram riscos carcinogênicos para ambas as condições analisadas (ambiente aberto e fechado) e ambos os receptores (trabalhadores e comercial). Os elementos benzeno, tolueno, etilbenzeno, o-xileno e m-xileno apresentaram risco não carcinogênico para ambientes fechados em solo subsuperficial contaminado. Em ambientes abertos, somente o xileno,m- não apresentou risco não carcinogênico. Para receptores trabalhadores, somente o benzeno apresentou risco não carcinogênico. Destaca-se a grande importância do gerenciamento de risco para posterior recuperação dessa área afetada.
\end{abstract}

Palavras-chave: risco à saúde humana; áreas contaminadas; risco carcinogênico; compostos orgânicos voláteis.

\begin{abstract}
The present study was an assessment and risk quantification of derivatives from oil spill at a refinery located in the Southeast of Brazil. The area presents subsurface soil and groundwater contaminated by petroleum hydrocarbons and other substances from the refinery. Inhalation exposure routes have been evaluated due to the presence of contaminants in the affected area. Risk quantification and hazard ratio were performed for each chemical substance, allowing to verify that, for exposure from contaminated groundwater, there was no carcinogenic and non-carcinogenic effect considering the inhalation route. As for exposure to contaminated subsurface soil, all the elements were detected as having the carcinogenic risk for both analyzed conditions (open and closed environments), and both receptors (worker and commercial). The elements Benzene, Toluene, Ethylbenzene, o-Xylene, and m-Xylene, have presented a non-carcinogenic risk for closed environments from contaminated subsurface soil. In open environments, only m-Xylene did not present non-carcinogenic risk. For the workers receptors, only Benzene has presented a non-carcinogenic risk. It might be highlighted the importance of risk management for the subsequent recovery of this affected area.
\end{abstract}

Keywords: risk to human health; contaminated areas; carcinogenic risk; volatile organic compounds.

\section{INTRODUÇÃO}

O petróleo é, por definição, um combustível fóssil líquido e oleoso, rico em hidrocarbonetos, principalmente alcanos (COSTA; JEREMIAS; MENEZES, 2015). Pela sua capacidade toxicológica, a indústria de petróleo é um dos ramos de maior potencial de impacto ambiental (CALDAS; VIANA; SANTOS, 2017). Em todas as fases de seu processo produtivo, desde o processo de extração, transporte, refino, até o consumo, a indústria petrolífera tem potencial para causar impactos sobre o ambiente e a saúde das populações, especialmente a saúde dos trabalhadores, no que diz respeito aos riscos específicos no ambiente de trabalho (HEIDERSCHEIDT et al., 2016).
Contendo centenas de hidrocarbonetos, o petróleo é uma mistura complexa, podendo ser dividido em saturado, aromático e orgânico polar. Os hidrocarbonetos saturados são subdivididos em alcanos de cadeias lineares, ramificadas ou ciclos alcanos, com vários números de anéis saturados e cadeias laterais. Os hidrocarbonetos aromáticos podem conter um ou mais anéis aromáticos, formando compostos monoaromáticos. Os compostos orgânicos polares são formados de heteroátomos polares (ABDEL-SHAFY; MANSOUR, 2016).

Os compostos de benzeno, tolueno, etilbenzeno e os xilenos -o-xileno, $\mathrm{m}$-xileno e p-xileno (BTEX) além de apresentarem extensa solubilidade em água

${ }^{1}$ Universidade Federal de Pelotas - Pelotas (RS), Brasil.

¿Universidade Federal do Rio Grande do Sul - Porto Alegre (RS), Brasil.

*Autora correspondente: carol_demarco@hotmail.com

Conflitos de interesse: os autores declaram não haver conflito de interesses.

Financiamento: nenhum.

Recebido: 30/11/2018 - Aceito: 13/11/2019 - Reg. ABES: 20180215 
(RENGARAJAN et al., 2015), são complexos e tóxicos, o que torna de grande importância os estudos de áreas contaminadas por combustíveis derivados de petróleo (ANDRADE; AUGUSTO; JARDIM, 2010). Esses compostos são nocivos tanto ao meio ambiente quanto ao ser humano, atuando como depressores do sistema nervoso central e apresentando toxicidade crônica mais significativa que os hidrocarbonetos alifáticos (LIMA et al., 2017).

Feisther et al. (2015) apontam que as propriedades físicas e químicas dos hidrocarbonetos influenciam a mobilidade da gasolina na água e no solo. Por sua solubilidade, esses compostos são utilizados como os principais indicadores de contaminação por combustíveis fósseis, uma vez que serão os primeiros a atingir o lençol freático (FREITAS et al., 2016).

A industrialização e os processos produtivos resultantes da extração e produção dos derivados de petróleo expõem cada vez mais um número relevante de trabalhadores a agentes químicos, com eventuais danos carcinogênicos e não carcinogênicos (LIMA et al., 2017). A preocupação com a saúde dos trabalhadores acabou tornando-se um tema importante no setor das políticas de saúde. Os operários que permanecem em exposição prolongada a esses agentes, mesmo que em pequenas quantidades, veem-se diante de possibilidades elevadas de agravos à saúde, que são de difícil percepção (D’ALASCIO et al., 2014). Muitas vezes os marcadores que podem diagnosticar esse tipo de agravo são desprezados (CARVALHO et al., 2017).

Segundo a Organização Pan-Americana da Saúde (OPAS, 2018), a avaliação de risco à saúde humana é um processo de levantamento e análise de informações ambientais e de saúde, mediante técnicas específicas, para subsidiar a tomada de decisão e a implantação, de maneira sistemática, de ações e de articulação intra e intersetorial, visando à promoção e proteção da saúde para melhorar as condições sociais e de vida das populações.

Quando uma área é impactada, existem metodologias de análise para o gerenciamento dos riscos que possibilitam a tomada de decisões. A American Society for Testing and Materials (ASTM) publicou a norma E1739-95, a qual aborda a metodologia "ação corretiva baseada no risco" (risk-based corrective action - RBCA) (ASTM, 1995), inicialmente aplicada a hidrocarbonetos e, posteriormente, ampliada para outras substâncias químicas (TAKEUCHI; ROISENBERG, 2009).

No Brasil, a metodologia para avaliação de riscos em áreas contaminadas foi elaborada em normas americanas (metodologia RBCA) e adaptada para o contexto brasileiro pela Companhia Ambiental do Estado de São Paulo (CETESB), sendo descrita no Manual de Gerenciamento de Áreas Contaminadas (CETESB, 2001). É considerado um procedimento de tomada de decisão para avaliação de risco e resposta a lançamentos de produtos químicos com base na proteção à saúde e ao meio ambiente (PORFÍRIO, 2014).

A metodologia RBCA permite estimar os riscos em uma área contaminada, em relação ao meio ambiente ou à saúde humana (PORFÍRIO, 2014). Proporciona, assim, uma gestão ambiental mais eficiente, visto que prioriza a disponibilização de recursos financeiros para locais com maiores problemáticas de contaminação (TAKEUCHI; ROISENBERG, 2009).

Segundo Endlich e Haus (2015), a via de ingresso é a maneira pela qual as substâncias e os compostos químicos de interesse entram em contato com a população exposta. Os meios pelos quais as substâncias podem introduzir-se no organismo dos receptores são considerados em função das vias de ingresso, levadas em conta na avaliação de risco à saúde humana em áreas contaminadas. São elas (ABNT, 2013): ingestão, inalação e contato dérmico.
Na exposição por inalação, via de ingresso selecionada para este estudo, os gases podem desprender-se diretamente do solo contaminado, atingindo pessoas tanto em ambiente externo (aberto) como em ambiente interno (fechado), ou da água durante o banho, considerando-se que a água da rede de distribuição pode ser contaminada pelo processo de permeação. A exposição humana geralmente ocorre em fábricas de produção e suas vizinhanças por contato dermal e inalação (CETESB, 2001). Destaca-se ainda que as outras vias de ingresso (dermal e ingestão) devem ser analisadas em futuros estudos.

Numerosos efeitos causados pela inalação do petróleo são atribuídos ao comportamento dos hidrocarbonetos voláteis, como o benzeno, n-hexano, n-pentano, tolueno e xilenos (CAIRNEY et al., 2002). De acordo com Oliveira et al. (2011), quando esses voláteis são inalados, uma pequena parcela é exalada no ar sem modificação, e o resto é convertido em metabólitos hidrofílicos antes de ser excretado na urina.

Diante disso, o objetivo deste estudo foi avaliar o risco à saúde humana de efeitos carcinogênicos e não carcinogênicos decorrentes da inalação de vapores de hidrocarbonetos de petróleo provenientes de solos contaminados, em uma refinaria na Região Sudeste do país.

\section{METODOLOGIA}

\section{Área de estudo}

O problema apresentado no presente trabalho consiste na avaliação da existência de risco e sua quantificação em casos de vazamentos de derivados do petróleo em uma refinaria localizada na Região Sudeste do Brasil, a qual terá seu nome preservado por razões éticas. Trabalho semelhante para essa área foi desenvolvido por Siqueira (2017) com solo contaminado por hidrocarbonetos de petróleo e outras substâncias em razão do funcionamento da refinaria. Constatou-se também a contaminação da água subterrânea. Assim, foram consideradas as vias de exposição por inalação aos contaminantes presentes nesses dois meios contaminados, quais sejam: inalação, em ambientes abertos, de vapores provenientes do solo subsuperficial; inalação, em ambientes fechados, de vapores provenientes do solo subsuperficial; inalação, em ambientes abertos, de vapores provenientes da água subterrânea; inalação, em ambientes fechados, de vapores provenientes da água subterrânea.

\section{Cálculo da dose de ingresso}

A dose de ingresso refere-se à quantificação dos compostos que potencialmente ingressaram no organismo exposto por uma determinada via de ingresso, considerando-se cada caminho de exposição identificado (CETESB, 2001). A quantificação baseia-se no critério denominado de exposição máxima razoável (EMR), o qual representa a máxima exposição razoavelmente esperada em um local, para cenários de uso atual e futuro (USEPA, 1989). A equação para a avaliação da exposição por inalação (obtenção das doses de entrada no corpo) é definida pela United States Environmental Protection Agency (USEPA, 1989) e está descrita na Equação 1.

$\mathrm{I}=\frac{\mathrm{C} \times \mathrm{CR} \times \mathrm{EF} \times \mathrm{ED}}{\mathrm{BW} \times \mathrm{AT}}$

Em que:

$\mathrm{C}=$ concentração do contaminante no $\operatorname{ar}\left(\mathrm{mg} \cdot \mathrm{L}^{-1}\right)$; 
$\mathrm{CR}=$ taxa de inalação $\left(\mathrm{m}^{3} \cdot \mathrm{dia}^{-1}\right)$;

$\mathrm{EF}=$ frequência da exposição $\left(\right.$ dias.ano $\left.{ }^{-1}\right)$;

$\mathrm{ED}=$ duração da exposição (anos);

$\mathrm{BW}=$ massa corpórea $(\mathrm{kg})$;

$\mathrm{AT}=$ tempo médio de avaliação da exposição (dias).

Os parâmetros gerais que foram utilizados para os cálculos das doses de ingresso estão apresentados na Tabela 1. Para este estudo, consideraram-se receptores dos tipos "comercial" e "trabalhadores eventuais", os quais estão expostos por diferentes períodos de tempo aos contaminantes analisados. Os receptores do tipo comercial referem-se aos que estão expostos durante todo o período de trabalho, enquanto os trabalhadores são os que têm exposição esporádica na área contaminada. Na Tabela 1, AT refere-se ao tempo médio de avaliação da exposição (dias); ED é a duração da exposição (anos); EF é a frequência da exposição (dias/ano); CR é a taxa de contato para um caminho de exposição ( $\mathrm{L} / \mathrm{dia}$ ou m³/dia); BW é a massa corpórea $(\mathrm{kg})$; TRi (individual) refere-se à meta de risco carcinogênico para cada contaminante; TRc (cumulativo) é a meta de risco carcinogênico para a soma de todos os contaminantes; THQ é a meta de quociente de perigo para cada contaminante; THI é a meta de quociente de perigo para a soma de todos os contaminantes (SIQUEIRA, 2017).

\section{Contaminantes presentes no solo e na água subterrânea}

As concentrações dos contaminantes no solo $\left(\mathrm{mg}^{\mathrm{kg}} \mathrm{kg}^{-1}\right)$ e na água subterrânea (mg.L $\mathrm{L}^{-1}$ ) observadas na área de estudo foram obtidas com base no estudo realizado por Siqueira (2017). Para o cálculo dos riscos de efeitos carcinogênicos e não carcinogênicos, foi necessário converter as concentrações presentes no solo e água subterrânea (origens) em concentrações estimadas no ar em ambientes abertos e fechados (destino). Para tal, foram utilizados os fatores descritos na Tabela 2. Os parâmetros utilizados para os cálculos dos fatores contidos na Tabela 2 estão apresentados nas Tabelas 3, 4 e 5 .

Tabela 1 - Parâmetros gerais utilizados nos cálculos das doses de ingresso por tipo de receptor.

\begin{tabular}{|c|c|c|c|c|}
\hline \multirow{2}{*}{ Parâmetro } & \multicolumn{2}{|c|}{ Carcinogênicos } & \multicolumn{2}{|c|}{ Não Carcinogênicos } \\
\hline & Comercial & Trabalhadores & Comercial & Trabalhadores \\
\hline AT (anos) & 68 & 68 & 25 & 1 \\
\hline ED (anos) & 25 & 1 & 25 & 1 \\
\hline EF (dias/ano) & 250 & 180 & 250 & 180 \\
\hline $\mathrm{CR}\left(\mathrm{m}^{3} / \mathrm{dia}\right)$ & 20 & 20 & 20 & 20 \\
\hline BW (kg) & 70 & 70 & 70 & 70 \\
\hline $\mathrm{TR}_{\mathrm{i}}$ (individual) & $1 \mathrm{E}-06$ & $1 E-06$ & - & - \\
\hline $\mathrm{TR}_{\mathrm{c}}$ (cumulativo) & $1 \mathrm{E}-05$ & $1 \mathrm{E}-\mathrm{O5}$ & - & - \\
\hline THQ (individual) & - & - & 1 & 1 \\
\hline THI (cumulativo) & - & - & 1 & 1 \\
\hline
\end{tabular}

AT: tempo médio de avaliação da exposição (dias); ED: duração da exposição (anos); EF: frequência da exposição (dias.ano'); CR: taxa de inalação ( $m^{3}$.dia'); BW: massa corpórea (kg); TR: meta de risco carcinogênico para cada contaminante; $\mathrm{TR}_{c}$ : meta de risco carcinogênico para a soma de todos os contaminantes; THQ: meta de quociente de perigo para cada contaminante; $\mathrm{THI}$ : meta de quociente de perigo para a soma de todos os contaminantes.

Fonte: Melo (2010).

\section{Riscos de câncer e quocientes de perigo}

Após o cálculo das concentrações de exposição no ar, o risco é calculado de distintas formas para os contaminantes carcinogênicos e não carcinogênicos. No caso dos carcinogênicos, a dose de exposição avaliada (I) é multiplicada por um fator de carcinogenicidade correspondente à via $n$ de ingresso no corpo por inalação, conforme Equação 2.

Risco $=\operatorname{In} \times \mathrm{SFi}_{i}$

Em que:

In $=$ dose de ingresso por via de contato ou caminho de exposição $n\left(\mathrm{mg} \cdot \mathrm{kg}^{-1} \cdot \mathrm{dia}^{-1}\right)$; $\mathrm{SF}_{\mathrm{i}}=$ fator de carcinogenicidade para inalação $\left(\mathrm{kg} \cdot\right.$ dia.mg $\left.{ }^{-1}\right)$.

No caso de contaminantes não carcinogênicos, a determinação do risco é dada pela razão entre a dose de ingresso de uma substância e a dose de referência, apresentada na Equação 3, denominada de quociente de perigo.

$\mathrm{HQ}=\mathrm{In} / \mathrm{RfDi}$

Em que:

$\mathrm{HQ}=$ quociente de perigo;

$\mathrm{RfDi}=$ dose de referência.

Caso esse quociente seja superior a $1(\mathrm{THQ}<1 \mathrm{e} \mathrm{THI}<1)$, isso quer dizer que a dose de exposição é maior que a dose de referência, ou seja, existe perigo de efeito tóxico à saúde humana. No caso de efeitos carcinogênicos, o risco de câncer deve ser menor que 1E-06 $\left(\mathrm{TR}_{\mathrm{i}}<1 \mathrm{E}-06 \mathrm{e} \mathrm{TR}_{\mathrm{c}}<1 \mathrm{E}-05\right)$.

Nos bancos de dados toxicológicos não constam os valores de RfDi e SFi, e eles apresentam somente URFi (fator de unidade de risco para inalação) e $R f C i$ (concentração de referência para inalação). Fatores de conversão para inalação são adotados seguindo critérios de ajustes, de acordo com a PRG - Region 9 (USEPA, 2009) conforme Equações 4 e 5.

$\operatorname{RfD}_{\mathrm{i}}\left[\frac{\mathrm{mg}}{\mathrm{kg}-\text { dia }}\right]=\operatorname{RfC}_{\mathrm{i}}\left(\frac{\mathrm{mg}}{\mathrm{m}^{3}}\right) \times \frac{20 \mathrm{~m}^{3}}{\text { dia }} \times \frac{1}{70 \mathrm{~kg}}$

$\mathrm{SF}_{\mathrm{i}}\left[\frac{\mathrm{kg}-\mathrm{dia}}{\mathrm{mg}}\right]=\mathrm{URF}_{\mathrm{i}}\left(\frac{\mathrm{m}^{3}}{\mathrm{mg}}\right) \times \frac{\mathrm{dia}}{20 \mathrm{~m}^{3}} \times 70 \mathrm{~kg}$

Em que:

$R f C_{\mathrm{i}}=$ concentração de referência para inalação $\left(\mathrm{mg} \cdot \mathrm{m}^{-3}\right)$;

$R f D i=$ dose de referência para inalação $\left(\mathrm{mg} \cdot \mathrm{kg}^{\left.-\mathrm{dia}^{-1}\right)}\right.$;

$S F_{\mathrm{i}}=$ fator de carcinogenicidade para inalação $\left(\mathrm{mg} \cdot \mathrm{kg}-\mathrm{dia}^{-1}\right)^{-1}$;

$U R F_{\mathrm{i}}=$ fator de unidade de risco para inalação $\left(\mathrm{mg} \cdot \mathrm{m}^{-3}\right)^{-1}$.

Como o risco individual de cada composto não é viável na prática, mas sim em sua totalidade (MELO, 2010), deve-se calcular também o risco da exposição total dos contaminantes simultaneamente. O risco total de câncer de exposição (para efeitos carcinogênicos) ou o índice de perigo (para efeitos não carcinogênicos) a todos os contaminantes simultaneamente é obtido pela soma dos riscos de exposições individuais.

A avaliação total dos contaminantes para todas as vias de exposição é calculada com recurso ao mesmo meio de contato com o receptor. Por exemplo, para 
saber qual é o risco (Equação 6) ou perigo de exposição total (Equação 7) de um contaminante para todas as vias possíveis de inalação, tem-se: (MELO, 2010):

Carcinogênico:

${ }_{\text {Risco }} \mathrm{ET}^{=} \sum$ Riscon $_{\mathrm{n}}$

Não carcinogênico:

$\mathrm{HI}_{\mathrm{ET}}=\sum \mathrm{HQn}_{\mathrm{n}}$

Em que:

Risco $_{\mathrm{ET}}=$ risco carcinogênico para exposição total;

Risco $_{\mathrm{n}}=$ risco carcinogênico para o caminho de exposição $\mathrm{n}$;
$H I_{\mathrm{ET}}=$ índice de perigo para exposição total;

$H I_{\mathrm{n}}=$ índice de perigo para o caminho de exposição n.

Destaca-se que alguns dos parâmetros utilizados nas equações apresentadas foram estimados e não obtidos experimentalmente. Os fatores de carcinogenicidade e as doses de referência são obtidos de testes de toxicidade em animais. Isso significa estimar o potencial que um determinando composto tem para provocar efeitos adversos em populações expostas, bem como obter uma estimativa da relação entre a extensão da exposição e o aumento na probabilidade de efeitos adversos, isto é, da relação dose-resposta.

Tabela 2 - Coeficientes de difusão e fatores de volatilização.

\begin{tabular}{|c|c|c|c|}
\hline Fator & Sigla & Equação & Definição \\
\hline $\begin{array}{l}\text { Coeficiente de } \\
\text { difusão efetiva }\end{array}$ & $D_{\text {eff }}$ & $D_{\mathrm{n}}^{\mathrm{eff}}=\mathrm{D}^{\mathrm{air}} \frac{\theta_{\mathrm{a}(\mathrm{n})}^{3.33}}{\theta_{\mathrm{t}(\mathrm{n})}^{2}}+\mathrm{D}^{\mathrm{wat}} \frac{1}{\mathrm{H}} \frac{\theta_{\mathrm{w}(\mathrm{n})}^{3.33}}{\theta_{\mathrm{t}(\mathrm{n})}^{2}}$ & $\begin{array}{l}\text { O coeficiente de difusão efetiva é um fator de } \\
\text { atenuação referente à transferência entre meios, } \\
\text { em que o transporte de compostos orgânicos } \\
\text { em uma matriz de solo é composto da fase } \\
\text { dissolvida da matriz do solo e da fase vapor. }\end{array}$ \\
\hline $\begin{array}{l}\text { Coeficiente de } \\
\text { difusão efetiva na } \\
\text { zona não saturada }\end{array}$ & $D_{s}^{\text {eff }}$ & $\mathrm{D}_{\mathrm{s}}^{\mathrm{eff}}\left[\frac{\mathrm{cm}^{2}}{\mathrm{~s}}\right]=\mathrm{D}^{\text {air }} \frac{\theta_{\mathrm{as}}^{3.33}}{\theta_{\mathrm{t}}^{2}}+\mathrm{D}^{\text {wat }} \frac{1}{\mathrm{H}} \frac{\theta_{\mathrm{ws}}^{3.33}}{\theta_{\mathrm{t}}^{2}}$ & $\begin{array}{l}\text { O coeficiente representa a difusão efetiva de } \\
\text { um composto orgânico através dos poros do } \\
\text { solo da zona não saturada. }\end{array}$ \\
\hline $\begin{array}{l}\text { Coeficiente de } \\
\text { difusão efetiva na } \\
\text { franja capilar }\end{array}$ & $\mathrm{D}_{\text {cap }}^{\text {eff }}$ & $D_{\text {cap }}^{\text {eff }}\left[\frac{\mathrm{cm}^{2}}{\mathrm{~s}}\right]=D^{\text {air }} \frac{\theta_{\text {acap }}^{3.33}}{\theta_{t}^{2}}+D^{\text {wat }} \frac{1}{h} \frac{\theta_{\text {acap }}^{3.33}}{\theta_{t}^{2}}$ & $\begin{array}{l}\text { É equivalente ao coeficiente de difusão efetiva } \\
\text { de um composto com os poros da matriz da } \\
\text { franja capilar. O conteúdo volumétrico de ar e } \\
\text { água da zona não saturada é substituído por } \\
\text { conteúdos volumétricos da franja capilar. }\end{array}$ \\
\hline $\begin{array}{l}\text { Coeficiente de } \\
\text { difusão efetiva acima } \\
\text { do nível da água }\end{array}$ & $D_{w s}{ }^{\text {eff }}$ & $D_{w s}^{e f f}\left[\frac{c^{2}}{s}\right]=\left(h_{c a p}+h_{v}\right)\left[\frac{h_{c a p}}{D_{w s}^{e f f}}+\frac{h_{v}}{D_{s}^{e f f}}\right]$ & $\begin{array}{l}\text { Está relacionado à difusão do composto de } \\
\text { interesse com a zona saturada e a franja capilar. }\end{array}$ \\
\hline $\begin{array}{l}\text { Fator de } \\
\text { volatilização em } \\
\text { ambientes abertos } \\
\text { provenientes do solo } \\
\text { subsuperficial }\end{array}$ & $V F_{\text {samb }}$ & $\begin{array}{l}V F_{\text {samb }}\left[\frac{\mathrm{mg} / \mathrm{m}^{3}-a r}{\mathrm{mg} / \mathrm{kg}-\mathrm{solo}]}\right]=\frac{C_{v, a m v}}{C_{s}} \\
=\frac{H \rho_{s}}{\left(\theta_{w s}+K_{d} \rho_{s}+H \theta_{a s}\right)\left(1+\frac{U_{a r} \delta_{a r} L_{s}}{D_{s}^{e f f} W}\right)} \times 10^{3} \frac{\mathrm{cm}^{3}-\mathrm{kg}}{\mathrm{m}^{3}-g}\end{array}$ & $\begin{array}{l}\text { Coeficiente de transferência das concentrações } \\
\text { do composto químico no solo subsuperficial } \\
\text { para o ambiente aberto em fase vapor. }\end{array}$ \\
\hline $\begin{array}{l}\text { Fator de volatilização } \\
\text { em ambientes } \\
\text { fechados } \\
\text { provenientes do solo } \\
\text { subsuperficial }\end{array}$ & $V F_{\text {sesp }}$ & $\begin{array}{l}V F_{\text {sesp }}\left[\frac{\mathrm{mg} / \mathrm{m}^{3}-a r}{\mathrm{mg} / \mathrm{kg}-\text { solo }}\right]=\frac{C_{v, e s p}}{C_{s}} \\
=\frac{\frac{H \rho_{s}}{\left[\theta_{w s}+K_{d} \rho_{s}+H \theta_{a s}\right]}\left[\frac{D_{s}^{\text {eff }} / L_{s}}{E R \times L_{B}}\right]}{1+\left[\frac{D_{s}^{e f f} / L_{s}}{E R+L_{B}}\right]+\left[\frac{D_{s}^{e f f} / L_{s}}{\left(D_{\text {crack }}^{\text {eff }} / L_{\text {crack }}\right) \eta}\right]} \times 10^{3} \frac{\mathrm{cm}^{3}-\mathrm{kg}}{\mathrm{m}^{3}-g}\end{array}$ & $\begin{array}{c}\text { Representa uma relação de transferência } \\
\text { das concentrações dos compostos no solo } \\
\text { subsuperficial para ambientes fechados em fase } \\
\text { vapor. }\end{array}$ \\
\hline $\begin{array}{l}\text { Fator de volatilização } \\
\text { em ambientes } \\
\text { fechados, } \\
\text { provenientes da } \\
\text { água subterrânea }\end{array}$ & $V F_{\text {wesp }}$ & $\begin{array}{l}V F_{w e s p}\left[\frac{m g / m^{3}-a r}{m g / L-s o l o}\right]=\frac{C_{v, e s p}}{C_{s}} \\
=\frac{H\left[\frac{D_{w s}^{e f f} / L_{g w}}{E R \times L_{B}}\right]}{1+\left[\frac{D_{w s}^{e f f} / L_{g w}}{E R \times L_{B}}\right]+\left[\frac{D_{w s}^{e f f} / L_{g w}}{\left(D_{\text {crack }}^{e f f} / L_{\text {crack }}\right) \eta}\right]} \times 10^{3} \frac{L}{m^{3}}\end{array}$ & $\begin{array}{c}\text { Relação entre as concentrações do composto } \\
\text { na fase vapor em ambientes fechados (destino) } \\
\text { e as concentrações dissolvidas na água } \\
\text { subterrânea (origem). }\end{array}$ \\
\hline
\end{tabular}

Fonte: Melo (2010). 
Geralmente, ao realizar uma avaliação de risco à saúde humana, os parâmetros de entrada dos modelos são definidos em termos da estimativa mais provável, o que pode trazer incertezas. Consequentemente, esses modelos não podem predizer ou estimar a incerteza do risco obtido, podendo influenciar na seleção da melhor alternativa de remediação (COPTY;

Tabela 3 - Parâmetros físicos dos meios contaminados.

\begin{tabular}{|c|c|c|}
\hline parâmetro & Sigla & Valor \\
\hline $\begin{array}{l}\text { Velocidade do ar na zona de mistura do ambiente } \\
\left.\text { aberto (cm.s } \mathrm{s}^{-1}\right)\end{array}$ & $U_{a r}$ & 100 \\
\hline Altura da zona de mistura do ambiente aberto $(\mathrm{cm})$ & $\delta_{a r}$ & 100 \\
\hline Profundidade do nível de água (hcap + hv) (cm) & $L_{g w}$ & 400 \\
\hline $\begin{array}{l}\text { Comprimento da pluma de fase dissolvida, paralela } \\
\text { ao fluxo }(\mathrm{cm})\end{array}$ & $W$ & 15.000 \\
\hline $\begin{array}{l}\text { Conteúdo volumétrico de ar na zona não saturada } \\
\left(\mathrm{cm}^{3} \text {-ar.cm }{ }^{3} \text {-solo-1) }\right.\end{array}$ & $\theta_{\text {as }}$ & 0,27 \\
\hline $\begin{array}{l}\text { Conteúdo volumétrico de água na zona não } \\
\text { saturada ( } \mathrm{cm}^{3} \text {-água.cm-solo') }\end{array}$ & $\theta_{w s}$ & 0,17 \\
\hline $\begin{array}{l}\text { Razão entre o volume do espaço fechado e a área } \\
\text { de infiltração (cm) }\end{array}$ & $L_{B}$ & 300 \\
\hline Espessura da fundação da construção (cm) & $L_{\text {crack }}$ & 15 \\
\hline $\begin{array}{l}\text { Profundidade do topo do solo subsuperficial } \\
\text { contaminado }(\mathrm{cm})\end{array}$ & $L_{S}$ & 400 \\
\hline $\begin{array}{l}\text { Fração da área da fundação com fendas } \\
\left.\text { ( } \mathrm{cm}^{2} \text {-fendas. } \mathrm{cm}^{2} \text {-área total' }{ }^{-1}\right)\end{array}$ & $\eta$ & 0,01 \\
\hline Fração de carbono orgânico & $f_{o c}$ & 0,01 \\
\hline Densidade do solo $\left(\mathrm{g} . \mathrm{cm}^{-3}\right)$ & $\rho_{S}$ & 1,36 \\
\hline Renovação do ar em espaços fechados (1.5'). & $E R$ & 2,3OE - O4 \\
\hline
\end{tabular}

Fonte: Melo (2010).
FINDIKAKIS, 2000). Em termos gerais, a incerteza representa a falta de conhecimento sobre fatores que afetam a exposição ou risco, enquanto a variabilidade emerge da heterogeneidade entre pessoas, lugares e tempo (variabilidade individual, espacial e temporal) (USEPA, 1997). A forma de lidar com incertezas e variabilidade é tentando reduzi-las ou quantificá-las, quando não for possível eliminá-las. Dessa maneira, enquanto a incerteza pode ser reduzida adquirindo-se maior quantidade de dados representativos do fenômeno ou empregando-se modelos não tão simplificados ou conservadores, a variabilidade sempre estará presente em virtude da natureza aleatória do fenômeno de contaminação.

Tabela 5 - Parâmetros toxicológicos dos contaminantes.

\begin{tabular}{|c|c|c|}
\hline Contaminante & $\operatorname{URF}_{i}\left(m^{3} . \mu g^{-1}\right)$ & $\operatorname{RfD}_{i}$ \\
\hline Benzo(a)antraceno & 8,80E - O5 & $1, \mathrm{OOE}+\mathrm{OO}$ \\
\hline Crisênio & 3,29E - O4 & 9,OOE - O7 \\
\hline Benzo(b)fluoranteno & 8,80E - O5 & $1, \mathrm{OOE}+\mathrm{OO}$ \\
\hline Benzo(k)fluoranteno & 8,80E - O6 & 1,OOE - 01 \\
\hline Benzo(a)pireno & 2,09E - O3 & $1,10 E+01$ \\
\hline Benzo(g,h,i)perileno & - & - \\
\hline Indeno(1,2,3,c,d)pireno & 8,80E - O5 & $1,10 E+O O$ \\
\hline Dibenzo(a,h)antraceno & - & 4,OOE - O1 \\
\hline Benzeno & 8,29E - 06 & 5,95E - O3 \\
\hline Tolueno & - & 4,0OE - O1 \\
\hline Etilbenzeno & & $1, \mathrm{OOE}+\mathrm{OO}$ \\
\hline Xileno, o- & - & 7,0OE - 01 \\
\hline Xileno, m- & - & 7,OOE - 01 \\
\hline
\end{tabular}

URF; fator de unidade de risco para inalação $\left(m^{3} \cdot \mu \mathrm{g}^{1}\right)$; RfD; dose de referência para inalação (mg.kg-dia').

Fonte: Melo (2010)

Tabela 4 - Parâmetros físicos dos contaminantes e concentração no solo e na água subterrânea.

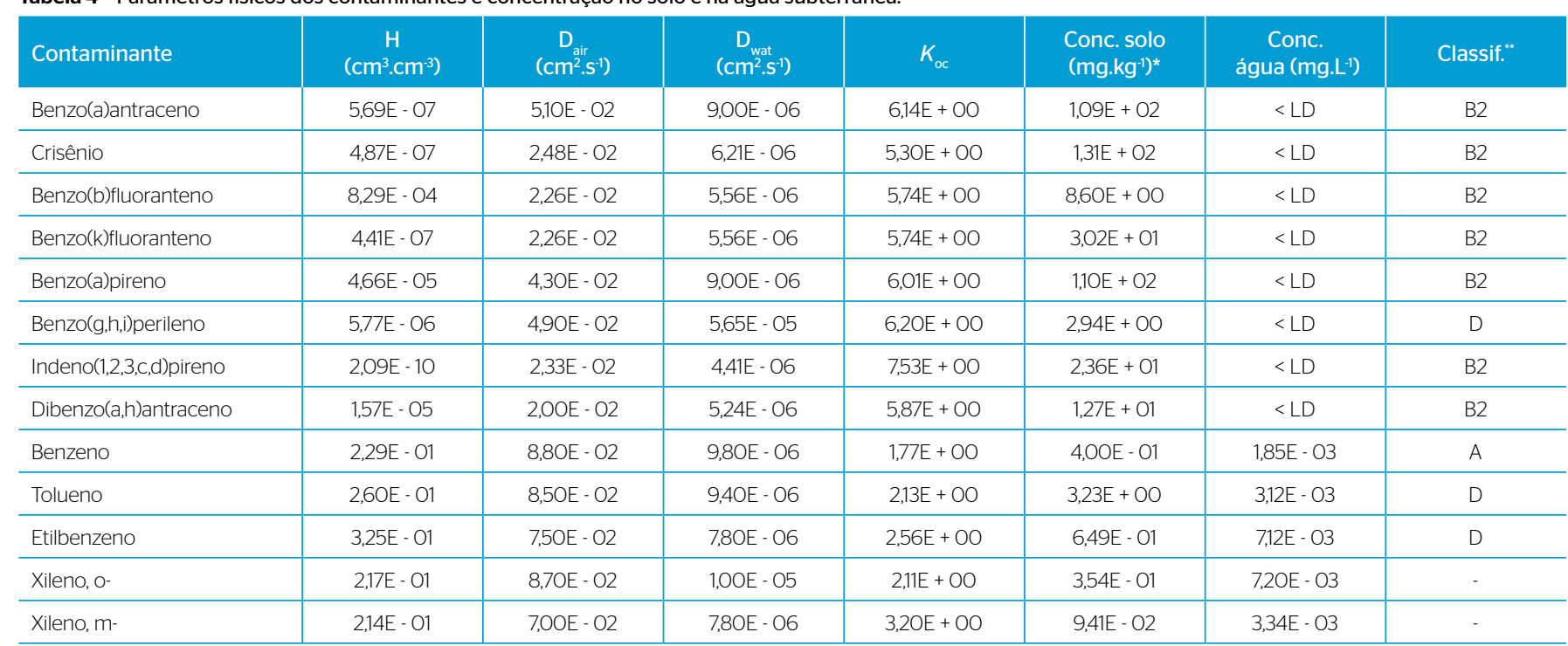

${ }^{* *}$ Classificação do potencial de carcinogenicidade conforme apresentado no banco de dados do software RBCA Tool Kit for Chemical Releases, versão 1.3a; < LD: menor que o limite detectável pelo método de amostragem; $\mathrm{H}$ : coeficiente da Lei de Henry ( $\mathrm{cm}^{3}$-água/ $\mathrm{cm}^{3}$-ar); $\mathrm{D}_{\text {arl }}$ : coeficiente de difusão do composto de interesse no ar ( $\left.\mathrm{cm}^{2} / \mathrm{s}\right) ; \mathrm{D}_{\text {wat }}$ coeficiente de difusão do composto de interesse na água $\left(\mathrm{cm}^{2} / \mathrm{s}\right)$.

Fonte: Melo (2010). 


\section{RESULTADOS E DISCUSSÃO}

\section{Dose inalada por via de exposição}

A Tabela 6 apresenta os valores de doses inaladas por meio de água subterrânea e solo subsuperficial contaminados, para ambientes abertos e fechados. Pode-se observar que a maior dose em água subterrânea corresponde ao etilbenzeno, que em ambientes fechados apresentou maior valor do que em ambientes abertos para efeitos não carcinogênicos, e aos receptores comerciais, os quais estão expostos com maior frequência.

$\mathrm{Na}$ análise das doses de benzeno, pode-se observar que em solo subsuperficial contaminado a taxa de inalação foi alta, sendo o benzeno um composto do Grupo 1, estabelecido pela International Agency for Research on Cancer (IARC, 2018) como carcinogênico para a saúde humana. Pode-se observar também que a dose de ingresso de benzeno foi maior para ambientes fechados, para receptores do tipo comercial. Destaca-se também o tolueno, o qual teve a maior concentração obtida em ambientes fechados e o crisênio em ambientes abertos.

Em relação aos demais componentes da gasolina e diesel, o benzeno possui maior solubilidade, o que o torna mais móvel no solo quando em períodos de maior fluxo de água (altas precipitações). Dessa forma, esse contaminante alcança longas distâncias, atingindo outros pontos de exposição, como fontes de água superficiais mais afastadas do ponto de contaminação de origem (SANTOS; VELASCO; LUZARDO, 2016).

Borillo (2015) avaliou as concentrações de BTEX em uma escola, avaliando os ambientes internos e externos, e constatou que o tolueno foi o composto mais abundante em $85,7 \%$ das amostras, com maiores concentrações ainda em ambientes internos. Cabe ressaltar que, para os compostos considerados

Tabela 6 - Doses inaladas por meio de água subterrânea e solo subsuperficial contaminados.

\begin{tabular}{|c|c|c|c|c|c|c|c|c|c|}
\hline \multirow{4}{*}{ Contaminante } & \multirow{4}{*}{ Conc. (mg..$\left.^{-1}\right)$} & \multicolumn{8}{|c|}{ Doses inaladas por meio de água subterrânea contaminada } \\
\hline & & \multicolumn{4}{|c|}{ Ambientes abertos } & \multicolumn{4}{|c|}{ Ambientes fechados } \\
\hline & & \multicolumn{2}{|c|}{ Carcinogênico } & \multicolumn{2}{|c|}{ Não carcinogênico } & \multicolumn{2}{|c|}{ Carcinogênico } & \multicolumn{2}{|c|}{ Não carcinogênico } \\
\hline & & $\begin{array}{c}\text { I } \\
\text { (comercial) } \\
\left(\mathrm{mg}^{\prime} \cdot \mathrm{kg}^{-1} \cdot \mathrm{dia}^{-1}\right)\end{array}$ & $\begin{array}{c}\text { I } \\
\text { (trabalh.) } \\
\left(\mathrm{mg}^{\mathrm{kg}} \mathrm{kg}^{-1} \cdot \mathrm{dia}^{-1}\right)\end{array}$ & $\begin{array}{c}\text { I } \\
\text { (comercial) } \\
\left(\mathrm{mg}^{-1} \mathrm{~kg}^{-1} \cdot \mathrm{dia}^{-1}\right)\end{array}$ & $\begin{array}{c}\text { I } \\
\text { (trabalh.) } \\
\left(\mathrm{mg}^{\mathrm{kg}} \mathrm{kg}^{-1} \cdot \mathrm{dia}^{-1}\right)\end{array}$ & $\begin{array}{c}\text { I } \\
\text { (comercial) } \\
\left(\mathrm{mg}^{\mathrm{kg}} \mathrm{cdia}^{-1}\right)\end{array}$ & $\begin{array}{c}\text { I } \\
\text { (trabalh.) } \\
\left(\mathrm{mg}^{\mathrm{kg}} \mathrm{kg}^{-1} \cdot \mathrm{dia}^{-1}\right)\end{array}$ & $\begin{array}{c}\text { I } \\
\text { (comercial) } \\
\left(\mathrm{mg}^{\prime} \mathrm{kg}^{-1} \cdot \mathrm{dia}^{-1}\right)\end{array}$ & $\begin{array}{c}\text { I } \\
\text { (trabalh.) } \\
\left(\mathrm{mg}^{-} \mathrm{kg}^{-1} \cdot \mathrm{dia}^{-1}\right)\end{array}$ \\
\hline Benzo(a)antraceno & $<L D$ & - & - & - & - & - & - & - & - \\
\hline Crisênio & $<L D$ & - & - & - & - & - & - & - & - \\
\hline Benzo(b)fluoranteno & $<L D$ & - & - & - & - & - & - & - & - \\
\hline Benzo(k)fluoranteno & $<L D$ & - & - & - & - & - & - & - & - \\
\hline Benzo(a)pireno & $<L D$ & - & - & - & - & - & - & - & - \\
\hline Benzo(g,h,i)perileno & $<L D$ & - & - & - & - & - & - & - & - \\
\hline Indeno(1,2,3,c,d)pireno & $<L D$ & - & - & - & - & - & - & - & - \\
\hline Dibenzo(a,h)antraceno & $<L D$ & - & - & - & - & & & & \\
\hline Benzeno & 1,85E - O3 & 2,07E - O7 & 1,49E - O9 & $5,62 \mathrm{E}-\mathrm{O} 7$ & 1,01E - O7 & $4,02 \mathrm{E}-\mathrm{O} 7$ & 2,89E - O9 & 1,09E - O6 & 1,97E - O7 \\
\hline Tolueno & $3,12 \mathrm{E}-\mathrm{O} 3$ & - & - & 1,04E - O6 & 1,87E - O7 & - & - & $2,02 \mathrm{E}-\mathrm{O} 6$ & 3,64E - O7 \\
\hline Etilbenzeno & $7,12 \mathrm{E}-\mathrm{O} 3$ & - & - & $2,62 \mathrm{E}-06$ & 4,71E - O7 & - & - & $5,09 E-06$ & 9,15E - O7 \\
\hline Xileno, o- & $7,2 \mathrm{OE}$ - 03 & - & - & $2,05 E-06$ & 3,69E - O7 & - & - & 3,98E - O6 & 7,17E - O7 \\
\hline Xileno, m- & 3,34E - O3 & - & - & 7,54E - O7 & 1,36E - O7 & - & - & 1,47E - O6 & $2,64 \mathrm{E}-\mathrm{O} 7$ \\
\hline Contaminante & Conc. (mg.kg $\left.{ }^{-1}\right)$ & \multicolumn{8}{|c|}{ Doses inaladas por meio de solo subsuperficial contaminado } \\
\hline Benzo(a)antraceno & $1,09 E+02$ & $5,41 E+01$ & 3,90E - 01 & - & - & 1,25E - 01 & 8,97E - O4 & - & - \\
\hline Crisênio & $1,31 E+O 2$ & $6,51 E+01$ & $4,68 E-01$ & - & - & 1,07E - 01 & 7,67E - O4 & - & - \\
\hline Benzo(b)fluoranteno & $8,60 \mathrm{E}+00$ & $4,27 \mathrm{E}+00$ & $3,08 \mathrm{E}-\mathrm{O} 2$ & - & - & 2,39E - 01 & $1,72 \mathrm{E}-\mathrm{O} 3$ & - & - \\
\hline Benzo(k)fluoranteno & $3, \mathrm{O} 2 \mathrm{E}+\mathrm{O} 1$ & $1,50 E+O 1$ & 1,08E - O1 & - & - & $2,14 \mathrm{E}-\mathrm{O} 2$ & $1,54 \mathrm{E}-\mathrm{O} 4$ & - & - \\
\hline Benzo(a)pireno & $1,10 E+02$ & $5,46 \mathrm{E}+01$ & 3,93E - 01 & - & - & $4,46 \mathrm{E}-\mathrm{O}$ & $3,21 \mathrm{E}-\mathrm{O} 3$ & - & - \\
\hline Benzo(g,h,i)perileno & $2,94 \mathrm{E}+\mathrm{OO}$ & - & - & - & - & - & - & - & - \\
\hline Indeno(1,2,3,c,d)pireno & $2,36 \mathrm{E}+\mathrm{O} 1$ & $1,17 E+01$ & $8,44 \mathrm{E}-\mathrm{O} 2$ & - & - & $1,18 \mathrm{E}-\mathrm{O} 2$ & 8,51E - O5 & - & - \\
\hline Dibenzo(a,h)antraceno & $1,27 E+01$ & $6,31 E+00$ & 4,54E - O2 & - & - & $1,43 \mathrm{E}-\mathrm{O} 2$ & 1,03E - O4 & - & - \\
\hline Benzeno & $4,00 E-01$ & 1,99E - 01 & $1,43 \mathrm{E}-\mathrm{O} 3$ & $5,40 E-O 1$ & 4,86E - 05 & $1,12 \mathrm{E}+\mathrm{O1}$ & $8,08 \mathrm{E}-\mathrm{O} 2$ & $3,05 E+01$ & $2,75 E-03$ \\
\hline Tolueno & $3,23 E+O O$ & - & - & $4,36 \mathrm{E}+\mathrm{OO}$ & 3,93E - O4 & - & - & $2,57 E+O 2$ & 2,31E - O2 \\
\hline Etilbenzeno & $6,49 E-01$ & - & - & 8,77E - 01 & 7,89E - 05 & - & - & $5,25 E+01$ & $4,72 E-O 3$ \\
\hline Xileno, o- & 3,54E - 01 & - & - & 4,78E - 01 & 4,30E - O5 & - & - & $2,52 \mathrm{E}+01$ & 2,26E - O3 \\
\hline Xileno, m- & $9,41 \mathrm{E}-\mathrm{O} 2$ & - & - & 1,27E - 01 & 1,14E - 05 & - & - & $5,03 E+O O$ & $4,53 E-04$ \\
\hline
\end{tabular}

Fonte: Elaborada pelos autores (2018) 
carcinogênicos, como o benzeno, devem ser avaliados os efeitos carcinogênicos e também não carcinogênicos. Por essa razão foram calculadas as doses para cada tipo de efeito.

\section{Riscos de câncer e quocientes de perigo por via de exposição}

Os resultados dos riscos de câncer e perigos decorrentes de água subterrânea e solo subsuperficial estão apresentados individualmente na Tabela 7. Os valores limites para vários compostos de interesse foram excedidos, tanto para efeitos tóxicos e carcinogênicos quanto para os tipos de receptores e vias de exposição (ambiente aberto e ambiente fechado).

Pode-se perceber que, em ambiente aberto, todos os compostos passíveis de detecção foram identificados como apresentando risco de câncer para os receptores comerciais, ou seja, apresentando risco superior a 1E-06: benzo(a) antraceno $($ risco $=1,67 \mathrm{E}+01)$, crisênio (risco $=7,49 \mathrm{E}+01)$, benzo(b)fluoranteno $($ risco $=1,32 \mathrm{E}+00)$, benzo $(\mathrm{k})$ fluoranteno $($ risco $=4,62 \mathrm{E}-01)$, benzo $(\mathrm{a})$ pireno $($ risco $=4,00 \mathrm{E}+02)$, indeno $(1,2,3, \mathrm{C}, \mathrm{D})$ pireno $($ risco $=3,61 \mathrm{E}+00)$, benzeno $($ risco $=5,76 \mathrm{E}-03)$ (Tabela 7$)$.

Considerando os receptores trabalhadores em ambiente aberto, verificou-se que os elementos que apresentaram risco de câncer à saúde humana foram: benzo(a)antraceno (risco $=1,20 \mathrm{E}-01)$, crisênio (risco $=5,39 \mathrm{E}-01)$, benzo(b) fluoranteno (risco $=9,47 \mathrm{E}-03)$, benzo $(\mathrm{k})$ fluoranteno $($ risco $=3,33 \mathrm{E}-03)$, benzo(a)pireno (risco $=2,88 \mathrm{E}+00)$, benzeno (risco $=4,15 \mathrm{E}-05)$.

Em ambientes fechados o tolueno apresentou valores acima do admissível para efeitos não carcinogênicos $(\mathrm{HQ}=2.25 \mathrm{E}+03)$. Para receptores comerciais (HQ limite $=1$ ), em ambientes abertos, também excedeu o valor aceitável $(\mathrm{HQ}=3.82 \mathrm{E}+01)$. Já o benzeno apresentou quociente de perigo de $\mathrm{HQ}=3.18 \mathrm{E}+02$ para ambientes abertos e $\mathrm{HQ}=1.80 \mathrm{E}+04$ para ambientes fechados.

Balthazar (2009), realizando diagnóstico geoambiental e avaliação de risco à saúde humana em terminal de abastecimento no munícipio de Sumaré (SP),

Tabela 7 - Riscos de câncer e quocientes de perigo para vias de exposição a água subterrânea e solo subsuperficial contaminados.

\begin{tabular}{|c|c|c|c|c|c|c|c|c|}
\hline \multirow[b]{2}{*}{ Contaminante } & \multicolumn{4}{|c|}{ Ambiente aberto } & \multicolumn{4}{|c|}{ Ambiente fechado } \\
\hline & $\begin{array}{l}\text { Risco (comercial) } \\
\quad\left(\mathrm{TR}_{\mathrm{1}}<1 \mathrm{e}-06\right)\end{array}$ & $\begin{array}{l}\text { Risco (trabalh.) } \\
\left(\mathrm{TR}_{\mathrm{l}}<1 \mathrm{e}-06\right)\end{array}$ & $\begin{array}{c}\mathrm{Hq} \\
(\text { comercial) } \\
(\mathrm{THQ}<1)\end{array}$ & $\begin{array}{c}\mathrm{Hq} \\
\text { (trabalh.) } \\
(\mathrm{THQ}<1)\end{array}$ & $\begin{array}{l}\text { Risco (comercial) } \\
\quad\left(\mathrm{TR}_{\mathrm{1}}<1 \mathrm{e}-06\right)\end{array}$ & $\begin{array}{l}\text { Risco (trabalh.) } \\
\left(\mathrm{TR}_{1}<1 \mathrm{e}-06^{6}\right)\end{array}$ & $\begin{array}{c}\mathrm{Hq} \\
(\text { comercial) } \\
(\mathrm{THQ}<1)\end{array}$ & $\begin{array}{c}\mathrm{Hq} \\
\text { (trabalh.) } \\
(\mathrm{THQ}<1)\end{array}$ \\
\hline & \multicolumn{8}{|c|}{ Riscos e quocientes de perigo para vias de exposição a água subterrânea contaminada } \\
\hline Benzo(a)antraceno & - & - & - & - & - & - & - & - \\
\hline Crisênio & - & - & - & - & - & - & - & - \\
\hline Benzo(b)fluoranteno & - & - & - & - & - & - & - & - \\
\hline Benzo(k)fluoranteno & - & - & - & - & - & - & - & - \\
\hline Benzo(a)pireno & - & - & - & - & - & - & - & - \\
\hline Benzo(g,h,i)perileno & - & - & - & - & - & - & - & - \\
\hline Indeno(1,2,3,c,d)pireno & - & - & - & - & - & - & - & - \\
\hline Dibenzo(a,h)antraceno & - & - & - & - & - & - & - & - \\
\hline Benzeno & 5,99E - O9 & 4,32E - 11 & 3,31E - O4 & $5,95 \mathrm{E}-\mathrm{O} 5$ & 1,17E - 08 & 8,39E - 11 & $6,43 E-04$ & 1,16E - O4 \\
\hline Tolueno & - & - & 9,09E - O6 & $1,64 \mathrm{E}-\mathrm{O} 6$ & - & - & 1,77E - O5 & 3,18E - O6 \\
\hline Etilbenzeno & - & - & 9,15E - O6 & 1,65E - O6 & - & - & 1,78E - 05 & $3,20 E-06$ \\
\hline Xileno, o- & - & - & 1,02E - O5 & $1,84 \mathrm{E}-\mathrm{O} 6$ & - & - & 1,99E - O5 & $3,58 E-06$ \\
\hline Xileno, m- & - & - & 3,77E - 06 & 6,79E - O7 & - & - & 7,33E - O6 & 1,32E - O6 \\
\hline Contaminante & \multicolumn{8}{|c|}{ Riscos de câncer e quocientes de perigo para vias de exposição a solo subsuperficial contaminado } \\
\hline Benzo(a)antraceno & $1,67 \mathrm{E}+01$ & $1,2 \mathrm{OE}-\mathrm{O} 1$ & - & - & $3,84 \mathrm{E}-\mathrm{O} 2$ & $2,76 \mathrm{E}-\mathrm{O} 4$ & - & - \\
\hline Crisênio & $7,49 E+01$ & $5,39 E-01$ & - & - & $1,23 E-01$ & 8,83E - O4 & - & - \\
\hline Benzo(b)fluoranteno & $1,32 \mathrm{E}+\mathrm{OO}$ & $9,47 E-03$ & - & - & 7,36E - O2 & 5,30E - O4 & - & - \\
\hline Benzo(k)fluoranteno & $4,62 E-O 1$ & 3,33E - O3 & - & - & $6,59 E-04$ & 4,75E - O6 & - & - \\
\hline Benzo(a)pireno & $4, \mathrm{OOE}+\mathrm{O} 2$ & $2,88 \mathrm{E}+\mathrm{OO}$ & - & - & $3,26 E+O O$ & 2,35E - O2 & - & - \\
\hline Benzo(g,h,i)perileno & - & - & - & - & - & - & - & - \\
\hline Indeno(1,2,3,c,d)pireno & $3,61 \mathrm{E}+\mathrm{OO}$ & 2,6OE - O2 & - & - & 3,64E - O3 & $2,62 \mathrm{E}-\mathrm{O} 5$ & - & - \\
\hline Dibenzo(a,h)antraceno & - & - & - & - & - & - & & \\
\hline Benzeno & $5,76 \mathrm{E}-\mathrm{O} 3$ & 4,15E - O5 & $3,18 \mathrm{E}+\mathrm{O} 2$ & $2.6 \mathrm{E}-\mathrm{O} 2$ & $3,26 \mathrm{E}-\mathrm{O1}$ & 2,34E - O3 & $1,80 \mathrm{E}+\mathrm{O} 4$ & $1,62 \mathrm{E}+\mathrm{OO}$ \\
\hline Tolueno & - & - & $3,82 \mathrm{E}+01$ & 3,44E - O3 & - & - & $2,25 E+O 3$ & 2,03E - 01 \\
\hline Etilbenzeno & - & - & 3,07E + OO & 2,76E - O4 & - & - & $1,84 \mathrm{E}+\mathrm{O} 2$ & 1,65E - O2 \\
\hline Xileno, o- & - & - & 2,39E + OO & 2,15E - O4 & - & - & $1,26 \mathrm{E}+\mathrm{O} 2$ & 1,13E - O2 \\
\hline Xileno, m- & - & - & 6,36E - 01 & 5,72E - O5 & - & - & $2,52 E+O 1$ & 2,26E - O3 \\
\hline
\end{tabular}

Fonte: elaborada pelos autores (2018). 
detectou valores de risco de efeitos carcinogênicos considerando exposição em ambiente aberto para o benzeno de 2,30E - 7. Esse valor é inferior aos encontrados neste estudo, tanto para receptores comerciais (5,76E -03) como para receptores trabalhadores $(4,15 \mathrm{E}-05)$. $\mathrm{O}$ autor também encontrou quociente de perigo em ambientes abertos inferior para o benzeno $(\mathrm{HQ}=9,3 \mathrm{E}-03)$, enquanto este estudo detectou valores de exposição para a classe comercial de $\mathrm{HQ}=3,18 \mathrm{E}$ +02 e $\mathrm{HQ}=2,86 \mathrm{E}-02$ para a classe de trabalhadores. O quociente de perigo encontrado pelo autor referente ao tolueno foi de $\mathrm{HQ}=1,3 \mathrm{E}-01$, inferior ao $\mathrm{HQ}$ detectado para a classe comercial neste estudo $(\mathrm{HQ}=3,82 \mathrm{E}+01)$ e superior ao dos receptores trabalhadores deste estudo (3,44E - 03).

Em relação ao Etilbenzeno, Balthazar (2009) detectou um quociente de perigo de 1,8E - 2, valor inferior ao HQ encontrado para os receptores comerciais deste estudo ( $\mathrm{HQ}=3,07 \mathrm{E}+00)$ e superior ao encontrado para os receptores trabalhadores ( $\mathrm{HQ}=2,76 \mathrm{E}-04)$.

Foi destacado pelo autor que, no local avaliado, excederam-se os limites de risco carcinogênico para inalação em ambiente fechado, com risco de 1,6E - 05 para o benzeno e quociente de perigo (HQ) de 9,0E + 00 para o tolueno. $\mathrm{O}$ etilbenzeno foi detectado em nível também acima do estabelecido, com HQ de $1,2 \mathrm{E}+00$.

Em relação aos riscos de câncer e quocientes de perigo para vias de exposição a água subterrânea contaminada, pode-se observar que nenhum risco de câncer foi menor $1 \mathrm{E}$ - 06 e que o quociente de perigo não foi superior a 1, o

Tabela 8 - Risco total de câncer e índice de perigo.

\begin{tabular}{|c|c|c|c|c|}
\hline Contaminante & $\begin{array}{l}\mathrm{Risco}_{\mathrm{et}} \\
\text { (comer- } \\
\text { cial) }\end{array}$ & $\begin{array}{l}\text { Risco }_{\text {et }} \\
\text { (trabalh.) }\end{array}$ & $\underset{\text { (comercial) }}{\mathrm{Hi}_{\mathrm{et}}}$ & $\begin{array}{c}\mathrm{Hi}_{\mathrm{et}} \\
\text { (trabalh.) }\end{array}$ \\
\hline Benzo(a)antraceno & $1,67 \mathrm{E}+01$ & $1,2 \mathrm{OE}-\mathrm{O}$ & - & - \\
\hline Crisênio & $7,50 \mathrm{E}+\mathrm{O1}$ & $5,40 \mathrm{E}-\mathrm{O} 1$ & - & - \\
\hline Benzo(b)fluoranteno & $1,39 E+O O$ & 1,OOE - O2 & - & - \\
\hline Benzo(k)fluoranteno & $4,63 E-01$ & 3,33E - O3 & - & - \\
\hline Benzo(a)pireno & $4,03 E+O 2$ & $2,90 \mathrm{E}+\mathrm{OO}$ & - & - \\
\hline Benzo(g,h,i)perileno & - & - & - & - \\
\hline Indeno(1,2,3,c,d)pireno & $3,61 E+O O$ & $2,60 \mathrm{E}-\mathrm{O} 2$ & - & - \\
\hline Dibenzo(a,h)antraceno & - & - & - & - \\
\hline Benzeno & 3,31E - 01 & 2,39E - O3 & $1,83 E+04$ & $1,64 \mathrm{E}+\mathrm{OO}$ \\
\hline Tolueno & - & - & $2,29 E+03$ & 2,06E - 01 \\
\hline Etilbenzeno & - & - & $1,87 E+02$ & $1,68 \mathrm{E}-\mathrm{O} 2$ \\
\hline Xileno, o- & - & - & $1,28 \mathrm{E}+02$ & $1,15 \mathrm{E}-\mathrm{O} 2$ \\
\hline Xileno, $\mathrm{m}$ & - & - & $2,58 \mathrm{E}+01$ & 2,32E - O3 \\
\hline
\end{tabular}

Fonte: Elaborada pelos autores (2018). que significa que não existe perigo de efeito carcinogênico nem efeito tóxico à saúde humana nas vias de exposição consideradas.

Nesse contexto, Santos, Velasco e Luzardo (2016) demonstraram que as concentrações de benzeno podem facilmente ser lixiviadas para a água subterrânea, porém essa substância possui menor propensão de volatilização para o ambiente externo. Dessa forma, o risco de toxicidade e de carcinogenicidade pela exposição às águas subterrâneas torna-se menor, uma vez que essa substância volatiliza menos. Isso corrobora os resultados deste trabalho, em que a via de exposição a água subterrânea contaminada não apresentou riscos para efeitos não carcinogênicos nem carcinogênicos para as classes consideradas.

A Tabela 8 apresenta os riscos totais de câncer de exposição para efeitos carcinogênicos e os índices de perigo para efeitos não carcinogênicos para todas as vias consideradas (somatório das quatro vias analisadas por contaminante). Pode-se observar que há risco carcinogênico para os receptores comerciais (Risco $>1 \mathrm{E}-05)$. Todas as substâncias apresentaram valores de risco maiores que o limite para os receptores trabalhadores e comercial, representando riscos carcinogênicos a essas classes.

Já com relação ao risco de efeitos não carcinogênicos, avaliado pelo índice de perigo, todas as substâncias analisadas apresentaram valores superiores ao limite $\left(\mathrm{HI}_{\mathrm{ET}}>1\right)$ para os receptores comerciais. Já para os receptores trabalhadores, apenas o benzeno representou risco de efeitos tóxicos à saúde humana.

Como descrito anteriormente, na prática, é mais viável a totalidade dos riscos e não sua individualidade (Tabela 9), uma vez que o ser humano inala todas as substâncias simultaneamente na mistura. Pode-se observar que o índice de perigo para exposição total, para solo subsuperficial em ambiente fechado, apresentou valor positivo que supera o limite aceitável.

No cálculo do risco carcinogênico total por via de exposição, as vias de inalação de vapores provenientes de solo subsuperficial em ambientes abertos e fechados apresentaram risco carcinogênico maior que $1 \mathrm{E}$ - 05, indicando que um indivíduo exposto a esses compostos tem uma chance adicional em $100 \mathrm{mil}$ de desenvolver câncer durante a sua vida. Quando o índice de perigo é menor que 1 , indica que a exposição predita é improvável de representar efeitos não carcinogênicos adversos à saúde.

Feldhaus (2013) avaliou o risco carcinogênico e o risco toxicológico em um posto de combustível, considerando o ambiente do empreendimento e a vizinhança e analisando o solo e a água. Os resultados obtidos foram, para inalação de vapores contaminados com risco carcinogênico, de 9,7E - 05, representando valor inferior ao limite estabelecido e, para risco toxicológico, de 3,0E +00 , superior ao limite de 1 . Já os efeitos na vizinhança também foram inferiores aos limites, de 6,3E - 07 e 2,1E - 02 para riscos carcinogênicos e toxicológicos, respectivamente.

Tabela 9 - Risco carcinogênico para exposição total e índice de perigo para exposição total.

\begin{tabular}{|c|c|c|c|c|}
\hline & Risco $_{n}$ & Risco $_{n}$ & $\mathrm{Hi}_{\mathrm{n}}$ & $\mathrm{Hi}_{\mathrm{n}}$ \\
\hline & Comercial & Trabalhador & Comercial & Trabalhador \\
\hline Inalação, em ambientes abertos, de vapores provenientes da água subterrânea & 5,99E - O9 & $4,32 \mathrm{E}-11$ & 3,63E - 04 & $6,53 \mathrm{E}-05$ \\
\hline Inalação, em ambientes fechados, de vapores provenientes da água subterrânea & 1,17E - O8 & $8,39 E-11$ & 7,05E - 04 & 1,27E - 04 \\
\hline Inalação, em ambientes abertos, de vapores provenientes do solo subsuperficial & 4,97E - O2 & $3,58 \mathrm{E}+00$ & $3,62 \mathrm{E}+\mathrm{O} 2$ & $3,26 \mathrm{E}-\mathrm{O} 2$ \\
\hline Inalação, em ambientes fechados, de vapores provenientes do solo subsuperficial & $3,83 \mathrm{E}+\mathrm{OO}$ & $2,75 \mathrm{E}-\mathrm{O} 2$ & $2,05 \mathrm{E}+04$ & $1,85 \mathrm{E}+\mathrm{OO}$ \\
\hline
\end{tabular}

Fonte: elaborada pelos autores (2018). 


\section{CONCLUSÕES}

A quantificação do risco e do quociente de perigo realizada para cada substância química permitiu verificar que, para a exposição a água subterrânea contaminada, não houve efeito carcinogênico e não carcinogênico para os compostos analisados por meio da via de inalação. Desse modo, não foi identificada a possibilidade de ocorrência de efeito adverso à saúde por essa via de exposição nas condições avaliadas.

Já para a exposição a solo subsuperficial contaminado, verificou-se que todos os elementos voláteis presentes em hidrocarbonetos de petróleo apresentaram risco carcinogênicos (benzo(a)antraceno, crisênio, benzo(b)fluoranteno, benzo(k)fluoranteno, benzo(a)pireno, indeno(1, 2, 3, C, D)pireno, benzeno) para ambas as condições analisadas (ambiente aberto e fechado) e ambos os receptores (trabalhadores e comercial). Os elementos benzeno, tolueno, etilbenzeno, xileno,o- e xileno,m- apresentaram risco não carcinogênico para ambientes fechados. Em ambientes abertos, somente o xileno,m- não apresentou risco não carcinogênico. Para receptores trabalhadores, somente o benzeno apresentou risco não carcinogênico.

Sugere-se a avaliação das outras rotas de exposição, como por exemplo a ingestão e o contato dermal, em futuros estudos, visando complementar a quantificação do risco e quociente de perigo. Destaca-se a grande importância do gerenciamento de risco para posterior recuperação dessa área afetada.

\section{CONTRIBUIÇÕES DOS AUTORES}

Cerbaro, D.: Escrita - Primeira Redação. Demarco, C. F.: Escrita - Revisão e Edição. Caballero, C. B.: Escrita - Revisão e Edição. Siqueira, T. M.: Conceituação, Curadoria de Dados, Supervisão.

\section{REFERÊNCIAS}

ABDEL-SHAFY, H.I.; MANSOUR, M.S.M. A review on polycyclic aromatic hydrocarbons: Source, environmental impact, effect on human health and remediation. Egyptian Journal of Petroleum, v. 25, n. 1, p. 107-123, 2016. https://doi.org/10.1016/j.ejpe.2015.03.011

AMERICAN SOCIETY FOR TESTING AND MATERIALS (ASTM). Risk-based corrective action applied at petroleum release sites. ASTM, 1995.

ANDRADE, J.A.; AUGUSTO, F; JARDIM, I.C.S.F. Biorremediação de solos contaminados por petróleo e seus derivados. Eclética Química, v. 35, n. 3, p. 17-43, 2010. https://doi.org/10.1590/S0100-46702010000300002

ASSOCIAÇÃO BRASILEIRA DE NORMAS TÉCNICAS (ABNT). NBR 16209: Avaliação de risco a saúde humana para fins de gerenciamento de áreas contaminadas. Rio de Janeiro: ABNT, 2013.

BALTHAZAR, R.B. Diagnóstico geoambiental e avaliação de risco à saúde humana em terminal de abastecimento no munícipio de Sumaré-SP. 77f. Trabalho de Conclusão de Curso (Graduação em Geologia) - Instituto de Geociências e Ciências Exatas, Universidade Estadual Paulista "Júlio de Mesquita Filho", Rio Claro, 2009.

BORILLO, G.C. Avaliação do Risco a Saúde causado por BTEX em escolas localizadas na região metropolitana de Curitiba-PR. 48f. Monografia (Especialização em Engenharia de Segurança do Trabalho) - PósGraduação em Engenharia de Segurança do Trabalho, Universidade Tecnológica Federal do Paraná, Curitiba, 2015

CAIRNEY, S.; MARUFF, P.; BURNS, C.; CURRIE, B. The neurobehavioural consequences of petrol (gasoline) sniffing. Neuroscience and Biobehavioural Reviews, v. 26, n. 1, p. 81-89, 2002. https://doi.org/10.1016/ S0149-7634(O1)00040-9

CALDAS, A.S.; VIANA, Z.C.V.; SANTOS, V.L.C.S. Fibras de Cocos nucifera como sorvente de petróleo em ambiente marinho. Acta Brasiliensis, v. 1, n. 1, p. 13-18, 2017. https://doi.org/10.22571/Actabra1120179

CARVALHO, L.V.B.; COSTA-AMARAL, I.C:; MATTOS, R.C.O.; LARENTIS, A.L. Exposição ocupacional a substâncias químicas, fatores socioeconômicos e Saúde do Trabalhador: uma visão integrada. Saúde em Debate, v. 41, n. esp. 2, p. 313-326, 2017. https://doi.org/10.1590/0103-11042017s226
COMPANHIA AMBIENTAL DO ESTADO DE SÃO PAULO (CETESB). Manual de gerenciamento de áreas contaminadas. $2^{\mathrm{a}}$ ed. São Paulo: Cetesb/GTZ, 2001. 595 p.

COPTY, N.K.; FINDIKAKIS, A.N. Quantitative Estimates of the Uncertainty in the Evaluation of Ground Water Remediation Schemes. Ground Water, v. 38, n. 1, p. 29-37, 2000. https://doi.org/10.1111/j.1745-6584.2000. tb00199.x

COSTA, P.D.; JEREMIAS, T.C.; MENEZES, C.T.B. Estudo de Turfa e Carvão Ativado como Adsorventes para remoção de hidrocarbonetos de meio aquoso. Tecnologia e Ambiente, v. 21, n. 1, p. 242-248, 2015. https://doi. org/10.18616/ta.v21iO.2244

D’ALASCIO, R.G.; MENEGALI, M.; BORNELLI, A.S.; MAGAJEWSKI, F. Sintomas relacionados à exposição ocupacional ao benzeno e hábitos ocupacionais em trabalhadores de postos de revenda de combustíveis a varejo na região sul de Santa Catarina. Revista Brasileira Medicina do Trabalho, v. 12, n. 1, p. 21-29, 2014.

ENDLICH, L.L.; HAUS, T.L. Estudo de caso para a comparação entre métodos de análise de risco: Rbca Tier 2 e análise de risco para postos de combustíveis, segundo a Cetesb. Memorial TCC Caderno da Graduação, v. 1, n. 1, p. 423-443, 2015

FEISTHER, V.A.; ULSON DE SOUZA, A.A.; TRIGUEROS, D.E.G.; MELLO, J.M.M.; OLIVEIRA, D.; SOUZA, S.M.A.G.U. Biodegradation kinetics of benzene, toluene and xylene compounds: microbial growth and evaluation of models. Bioprocess and Biosystems Engineering, v. 38, p. 1233-1241, 2015. https://doi.org/10.1007/s00449-015-1364-O

FREITAS, E.V.C.; BARRETO, F.M.S.; ALENCAR NETO, M.F.; CAVALCANTE, R.M. Avaliação do uso da cromatografia gasosa para detecção de hidrocarbonetos monoaromáticos na água subterrânea na Região Norte do município de Fortaleza (CE). Águas Subterrâneas, v. 30, n. 2, p. 289-305, 2016. https://doi.org/10.14295/ras.v30i2.28

HEIDERSCHEIDT, D.; PEREIRA, J.; BURGHARDT, J.E.; SILVA, L.A.; OLIVEIRA, S.C. Conceitos aplicados à poluição do solo decorrente do derrame de petróleo e seus derivados. Revista Maiêutica, v. 4, n. 1, p. 7-14, 2016. 
INTERNATIONAL AGENCY FOR RESEARCH ON CANCER (IARC). Agents Classified by the IARC Monographs, v. 1-123. IARC. Disponivel em: http:// monographs.iarc.fr/ENG/Classification/ClassificationsAlphaOrder.pdf. Acesso em: 6 abr. 2018.

LIMA, S.D.; OLIVEIRA, A.F.; GOLIN, R.; CAIXETA, D.S.; LIMA, Z.M.; MORAIS, E.B. Gerenciamento de áreas contaminadas por postos de combustíveis em Cuiabá, Mato Grosso, Brasil. Revista Ambiente \& Água, v. 12, n. 2, p. 299-315, 2017. https://doi.org/10.4136/ambi-agua.1872

MELO, T.M. Soluções analiticas para transporte de hidrocarbonetos de petróleo em água subterrânea: avaliação determinística e probabilística do risco à saúde humana. 154f. Dissertação (Mestrado em Recursos Hídricos e Saneamento Ambiental) - Instituto de Pesquisas Hidráulicas, Universidade Federal do Rio Grande do Sul, Porto Alegre, 2010.

OLIVEIRA, N.M.; BENTO, F.M.; CAMARGO, F.A.; KNORST, A.J.; SANTOS, A.L.; PIZZOLATO, T.M.; PERALBA, M.C. Biodegradation of commercial gasoline (24\% ethanol added) in liquid médium by microorganisms isolated from a landfarming site. Journal of Environmental Science and Health Part A, v. 46, n. 1, p. 86-96, 2011. https://doi.org/10.1080/10934529.2011.526909

ORGANIZAÇÃO PAN-AMERICANA DA SAÚDE (OPAS). Avaliação de RisCo à Saúde Humana. OPAS. Disponível em: https://www.paho.org/bra/index. php?option=com_content\&view=article $\&$ id=375:avaliacao-de-risco-asaude-humana\&ltemid=768. Acesso em: 6 abr. 2018.

PORFÍRIO, B. Avaliação de risco do aterro controlado de Morretes - PR usando a metodologia RBCA. 132f. Dissertação (Mestrado Profissional em Gestão Ambiental) - Universidade Positivo, Curitiba, 2014.
RENGARAJAN, T.; RAJENDRAN, P.; NANDAKUMAR, N.; LOKESHKUMAR, B.; RAJENDRAN, R.; NISHIGAKI, I. Exposure to polycyclic aromatic hydrocarbons with special focus on cancer. Asian Pacific Journal of Tropical Biomedicine, v. 5, n. 3, p. 182-189, 2015. https://doi.org/10.1016/S2221-1691(15)30003-4

SANTOS, A.S.; VELASCO, F.C.G.; LUZARDO, F.H.M. Avaliação da exposição ao benzeno em jazida de areia silicosa: cálculo do risco toxicológico. Ambiência, Guarapuava, v. 12, n. 1, p. 165-185, 2016. https://doi. org/10.5935/ambiencia.2016.01.10

SIQUEIRA, T.M. Emprego de fontes de contaminação de diferentes geometrias nas estimativas do risco à saúde humana devido à ingestão de água subterrânea contaminada. Revista Brasileira de Ciências Ambientais, n. 43, p. 85-100, 2017. https://doi.org/10.5327/Z2176-947820170113

TAKEUCHI, E.; ROISENBERG, A. Definição de níveis aceitáveis baseados no risco para hidrocarbonetos no Município de Porto Alegre segundo a metodologia RBCA. Engenharia Sanitária e Ambiental, v. 14, n. 4, p. 479-488, 2009. https://doi.org/10.1590/S1413-41522009000400008

UNITED STATES ENVIRONMENTAL PROTECTION AGENCY (USEPA). About EPA Region 4 (Southeast). Washington, D.C.: Usepa, 2009. Disponível em: http:// www.epa.gov/region4/waste/npl/nplnc/aberdnnc.htm. Acesso em: 10 mar. 2018.

UNITEDSTATES ENVIRONMENTAL PROTECTION AGENCY (USEPA). EXpOSURe Factors Handbook, EPA/600/P-95/OO2Fa. Washington, D.C.: Usepa, 1997.

UNITED STATES ENVIRONMENTAL PROTECTION AGENCY (USEPA). RisK assessment guidance for superfund: human health evaluation manual (Part A). Washington, D.C.: Usepa, 1989. v. 1. 